\title{
Dynamical generalization of a solvable family of two-electron model atoms with general interparticle repulsion
}

\author{
T. A. Niehaus, ${ }^{1,2}$ S. Suhai, ${ }^{2}$ and N. H. March ${ }^{3}$ \\ ${ }^{1}$ Bremen Center for Computational Materials Science, \\ University of Bremen, D-28359 Bremen, Germany \\ ${ }^{2}$ German Cancer Research Center, Dept. Molecular \\ Biophysics, D-69120 Heidelberg, Germany \\ ${ }^{3}$ Oxford University, Oxford, England
}

(Dated: November 24, 2018)

\begin{abstract}
Holas, Howard and March [Phys. Lett. A 310, 451 (2003)] have obtained analytic solutions for ground-state properties of a whole family of two-electron spin-compensated harmonically confined model atoms whose different members are characterized by a specific interparticle potential energy $\mathrm{u}\left(r_{12}\right)$. Here, we make a start on the dynamic generalization of the harmonic external potential, the motivation being the serious criticism levelled recently against the foundations of time-dependent density-functional theory (e.g. [J. Schirmer and A. Dreuw, Phys. Rev. A 75, 022513 (2007)]). In this context, we derive a simplified expression for the time-dependent electron density for arbitrary interparticle interaction, which is fully determined by an one-dimensional non-interacting Hamiltonian. Moreover, a closed solution for the momentum space density in the Moshinsky model is obtained.
\end{abstract}

PACS numbers: $31.15 . \mathrm{Ew}, 31.25 .-\mathrm{v}, 31.70 . \mathrm{Hq}$ 


\section{BACKGROUND AND OUTLINE}

A family of two-electron spin-compensated harmonically confined model atoms has recently been proposed and studied by Holas, Howard and March [1] (referred to below as HHM). Each member of this family is characterized by a specific interparticle potential energy $u\left(\left|\mathbf{r}_{1}-\mathbf{r}_{2}\right|\right)$. HHM showed that the ground-state spatial wave function $\Psi\left(\mathbf{r}_{1}, \mathbf{r}_{2}\right)$ then separated in centre-of-mass (c) and relative motion (b) coordinates defined by

$$
\mathbf{c}=\frac{\mathbf{r}_{1}+\mathbf{r}_{2}}{2} \quad, \quad \mathbf{b}=\mathbf{r}_{1}-\mathbf{r}_{2}
$$

to yield

$$
\Psi\left(\mathbf{r}_{1}, \mathbf{r}_{2}\right)=\psi_{\mathrm{CM}}(\mathbf{c}) \psi_{\mathrm{RM}}(\mathbf{b})
$$

The centre-of-mass term $\psi_{\mathrm{CM}}$ is determined once for all as a Gaussian function. Naturally the relative motion part $\psi_{\mathrm{RM}}$ entering Eq. (2), involves the interparticle repulsion $u(b)$, but only through an effective one-body potential energy $V_{\text {eff }}(b)$ given by (here and in the following atomic units are used $\left.\left[\hbar, e, m_{e}=1\right]\right)$

$$
V_{\mathrm{eff}}(b)=\frac{1}{4} \omega_{0}^{2} b^{2}+u(b)
$$

which must then be inserted in a one-particle Schrödinger equation to solve for the relative motion wave function $\psi_{\mathrm{RM}}$. Analytic solutions exist for a number of choices of $u(b)$ in Eq. (3), including harmonic [2], Coulombic [3] and inverse square law [4, 5].

As to the motivation for the dynamic generalization of the HHM static family of solutions in Ref. [1] above, we cite the recent criticism of Schirmer and Dreuw [6] that lies at the foundations of time-dependent density-functional theory (TDDFT); a very popular approximate route for current calculations of electronic excitation energies in atoms and molecules [7]. Because of such criticism, it seemed the more important to broaden considerably the class of available exact time-dependent analytical treatments. In that context, we must note here for Coulombic interaction in a two-electron model with harmonic confinement [8], the study of D'Amico and Vignale [9] in which the harmonic confinement is generalized to be time-dependent, the dimensionality of the problem these authors considered being reduced from three in the so-called Hookean atom [3, 8] to two.

Our objective here is to effect a related generalization of the whole family of two-electron model atoms considered by HHM to time-dependent theory. We therefore keep the discussion 
as general as possible and turn to specific forms of the interparticle interaction only in analytically solvable cases. The resulting expressions could serve as a convenient starting point for a simplified numerical treatment, which is however outside the scope of this work.

\section{TIME PROPAGATION OF HARMONICALLY CONFINED TWO-ELECTRON MODEL ATOMS}

We wish to solve the Schrödinger equation

$$
i \frac{\partial}{\partial t} \Psi=\hat{H} \Psi
$$

Here we take the model which is a time-dependent generalization of the study of HHM. Thus , the Hamiltonian operator assumed has the form

$$
\hat{H}=-\frac{1}{2}\left(\nabla_{r_{1}}^{2}+\nabla_{r_{2}}^{2}\right)+\frac{1}{2} \omega_{0}^{2}(t)\left(r_{1}^{2}+r_{2}^{2}\right)+u\left(\left|\mathbf{r}_{\mathbf{1}}-\mathbf{r}_{\mathbf{2}}\right|\right) .
$$

The time-dependent external potential involving $\omega_{0}^{2}(t)$ drives the system from its ground state $\Psi_{0}$ at $\mathrm{t}=0$ to a general time-dependent state $\Psi\left(\mathbf{r}_{1}, \mathbf{r}_{2}, \mathbf{t}\right)$ at time t. Following HHM we use centre-of-mass vector $\mathbf{c}$ and relative motion vector $\mathbf{b}$ in Eq. (1), to find

$$
\begin{aligned}
i \frac{\partial}{\partial t} \Psi(\mathbf{b}, \mathbf{c}, t)= & \\
& {\left[-\nabla_{\mathbf{b}}^{2}-\frac{1}{4} \nabla_{\mathbf{c}}^{2}+\frac{1}{4} \omega_{0}^{2}(t)\left(b^{2}+4 c^{2}\right)+u(b)\right] \Psi(\mathbf{b}, \mathbf{c}, t) . }
\end{aligned}
$$

With the help of the product ansatz

$$
\Psi(\mathbf{b}, \mathbf{c}, t)=\psi_{\mathrm{CM}}(\mathbf{c}, t) \psi_{\mathrm{RM}}(\mathbf{b}, t)
$$

Eq. (6) is without loss of generality readily separated into relative motion and centre-of-mass channels according to

$$
\begin{aligned}
i & \frac{\partial}{\partial t} \psi_{\mathrm{CM}}(\mathbf{c}, t)= \\
& {\left[-\frac{1}{2 m_{\mathrm{cm}}} \nabla_{\mathbf{c}}^{2}+\frac{m_{\mathrm{cm}}}{2} \omega_{0}^{2}(t) c^{2}\right] \psi_{\mathrm{CM}}(\mathbf{c}, t) } \\
i & \frac{\partial}{\partial t} \psi_{\mathrm{RM}}(\mathbf{b}, t)= \\
& {\left[-\frac{1}{2 m_{\mathrm{rm}}} \nabla_{\mathbf{b}}^{2}+\frac{m_{\mathrm{rm}}}{2} \omega_{0}^{2}(t) b^{2}+u(b)\right] \psi_{\mathrm{RM}}(\mathbf{b}, t), }
\end{aligned}
$$


with effective masses $m_{\mathrm{cm}}=2$ and $m_{\mathrm{rm}}=1 / 2$. It should be noted, that any (time-dependent) separation constant would give rise to phase factors of opposite sign in the RM and CM wave functions, which would then cancel in the formation of the total wave function and is hence neglected.

\section{A. Solution of the CM problem in two and three dimensions}

Since the centre-of-mass Hamiltonian does not depend on the interaction potential $u(b)$, the CM system be solved once and for all. In two dimensions this has already been accomplished by D'Amico and Vignale [9] for an arbitrary time dependence of $\omega_{0}(t)$. Their result for the CM wave function reads

$$
\psi^{\mathrm{CM}, 2 \mathrm{D}}(\mathbf{c}, t)=\sum_{n, m} c_{n m} \chi_{n m}(c, t) \Theta_{m}(\theta),
$$

where the fact that the Hamiltonian does not depend on the centre-of-mass angular variable $\theta$ was used to separate the wave function into an angular part characterized by the quantum number $m$

$$
\Theta_{m}(\theta)=\frac{1}{\sqrt{2 \pi}} e^{-i m \theta}
$$

and a radial part

$$
\chi_{n m}(c, t)=A(t) c^{m} e^{B(t) c^{2}} L_{n}^{m}\left[C(t) c^{2}\right]
$$

which involves the generalized Laguerre polynomials $L_{n}^{m}$ and the purely time-dependent functions [10]

$$
\begin{aligned}
A(t) & =\sqrt{\frac{n ! 2}{(n+m) !}}\left[m_{\mathrm{cm}} \dot{\phi}(t)\right]^{\frac{m+1}{2}} \\
& \times e^{-i(2 n+m+1)[\phi(t)-\phi(0)]} \\
B(t) & =-\frac{m_{\mathrm{cm}}}{2}\left[\dot{\phi}(t)-i \frac{d \ln |X(t)|}{d t}\right] \\
C(t) & =m_{\mathrm{cm}} \dot{\phi}(t) .
\end{aligned}
$$

The complex functions $X(t)$

$$
X(t)=|X(t)| e^{i \phi(t)} \quad \text { with } \quad \dot{\phi}(t)>0,
$$

are solutions to the equation of motion for the classical harmonic oscillator

$$
\ddot{X}=-\omega_{0}^{2}(t) X(t)
$$


which can be solved once an explicit form of $\omega_{0}^{2}(t)$ is chosen. Remarkably, the time propagation of the harmonically confined quantum system is fully determined by its classical analogue.

As a new result, we compute in the following the CM wave function for the general case of three dimensions. The derivation turns out to be straightforward and parallels the 2D case with minor modifications. To start with, we write the state $\psi_{n l m}^{\mathrm{CM}, 3 \mathrm{D}}$ with main quantum number $n$, angular momentum $l$ and magnetic quantum number $m$ as a product of a radial part $\tilde{\chi}_{n l}$ and spherical harmonics $Y_{l m}$ :

$$
\psi_{n l m}^{\mathrm{CM}, 3 \mathrm{D}}(\mathbf{c}, t)=\tilde{\chi}_{n l}(c, t) Y_{l m}(\theta, \phi) .
$$

The more general case in which the system is not in one of its eigenstates at $t=0$ can be handled easily according to Eq. (10), since the expansion coefficients do not depend on time. Notwithstanding, the ansatz Eq. (18) fully allows for excitations to eigenstates with different main quantum number due to the time dependent potential realized by $\omega_{0}(t)$.

Proceeding, the radial equation of motion takes the following form:

$$
i \frac{\partial}{\partial t} \tilde{\chi}_{n l}(c, t)=\left[-\frac{1}{2 m_{\mathrm{cm}}} \frac{\partial^{2}}{\partial c^{2}}-\frac{1}{m_{\mathrm{cm}} c} \frac{\partial}{\partial c}+\frac{l(l+1)}{2 m_{\mathrm{cm}} c^{2}}+\frac{1}{2} m_{\mathrm{cm}} \omega_{0}^{2}(t) c^{2}\right] \tilde{\chi}_{n l}(c, t),
$$

which we solve using a time-dependent generalization of the well known result for the isotropic harmonic oscillator in the ground state

$$
\tilde{\chi}_{n l}(c, t)=\tilde{A}(t) c^{l} e^{\tilde{B}(t) c^{2}} L_{\frac{1}{2}(n-l)}^{l+1 / 2}\left[\tilde{C}(t) c^{2}\right] .
$$

Inserting Eq. (20) into Eq. (19) and taking advantage of the defining differential equation of the associated Laguerre polynomials, we obtain after some algebra the following expressions for the functions $\tilde{A}, \tilde{B}$ and $\tilde{C}$ :

$$
\begin{aligned}
& i \dot{\tilde{A}}+\frac{(2 l+3)}{m_{\mathrm{cm}}} \tilde{A} \tilde{B}-\frac{(n-l)}{m_{\mathrm{cm}}} \tilde{A} \tilde{C}=0 \\
& i \dot{\tilde{B}}+\frac{2}{m_{\mathrm{cm}}} \tilde{B}^{2}-\frac{1}{2} m_{\mathrm{cm}} \omega_{0}^{2}(t)=0 \\
& i \dot{\tilde{C}}+\frac{4}{m_{\mathrm{cm}}} \tilde{B} \tilde{C}+\frac{2}{m_{\mathrm{cm}}} \tilde{C}^{2}=0 .
\end{aligned}
$$

Equations (21) to (23) are (besides different prefactors) completely equivalent to equations A4 to A6 in the mentioned work of D'Amico and Vignale, which allows us to write down 
the radial solution in three dimensions immediately:

$$
\begin{aligned}
\tilde{\chi}_{n l}(c, t)=\sqrt{\frac{2^{n+l+2} m_{\mathrm{cm}}^{l+\frac{3}{2}}\left[\frac{1}{2}(n-l)\right] !\left[\frac{1}{2}(n+l)\right] !}{\sqrt{\pi}(n+l+1) !}} & \times \\
{[\dot{\phi}(t)]^{\frac{2 l+3}{4}} \exp \left[-\frac{1}{2} m_{\mathrm{cm}}(\dot{\phi}(t)\right.} & \left.\left.-i \frac{d \ln |X(t)|}{d t}\right) c^{2}\right] \times \\
& e^{-i\left(n+\frac{3}{2}\right)[\phi(t)-\phi(0)]} c^{l} L_{\frac{1}{2}(n-l)}^{l+1 / 2}\left[m_{\mathrm{cm}} \dot{\phi}(t) c^{2}\right] .
\end{aligned}
$$

\section{B. Time dependent electron density for general interparticle interaction}

Having solved the problem for the CM system we now turn to an evaluation of the timedependent electron density $n(\mathbf{r}, t)$ for the special but important case of a system that is in its ground state at $t=0$. The square modulus of the CM wave function then reduces to a simple Gaussian function

$$
\left|\psi_{000}^{\mathrm{CM}, 3 \mathrm{D}}(\mathbf{c}, t)\right|^{2}=\frac{1}{a_{\mathrm{CM}}^{3}(t) \pi^{3 / 2}} \exp \left(-\frac{c^{2}}{a_{\mathrm{CM}}^{2}(t)}\right),
$$

where the time-dependence is fully governed by the characteristic length scale $a_{\mathrm{CM}}(t)$ of the oscillator:

$$
a_{\mathrm{CM}}(t)=\frac{1}{m_{\mathrm{cm}} \dot{\phi}(t)} .
$$

Since the potential $u(b)$ depends on the interparticle distance only, also the relative motion wave function $\psi^{R M, 3 D}$ can once again be split into angular and radial parts according to

$$
\psi_{n l m}^{\mathrm{RM}, 3 \mathrm{D}}(\mathbf{b}, t)=\tilde{\zeta}_{n l}(b, t) Y_{l m}(\theta, \phi),
$$

where $\tilde{\zeta}_{n l}(b, t)$ needs in general to be determined by time-propagation in one spatial dimension.

For the mentioned special case the electron density is given by

$$
\begin{aligned}
& n\left(\mathbf{r}_{1}, t\right)= \\
& 2 \int\left|\psi_{000}^{\mathrm{CM}, 3 \mathrm{D}}\left(\frac{1}{2}\left(\mathbf{r}_{\mathbf{1}}+\mathbf{r}_{\mathbf{2}}\right), t\right) \psi_{000}^{\mathrm{RM}, 3 \mathrm{D}}\left(\mathbf{r}_{\mathbf{1}}-\mathbf{r}_{\mathbf{2}}, t\right)\right|^{2} d \mathbf{r}_{2},
\end{aligned}
$$

which can be considerably simplified using Eq. (25) and (27). After evaluation of the angular 
integrations and substitution of $\mathbf{y}=\left(\mathbf{r}_{1}-\mathbf{r}_{\mathbf{2}}\right) / a_{\mathrm{CM}}$ for $\mathbf{r}_{\mathbf{2}}$, we arrive at

$$
\begin{aligned}
n\left(\mathbf{r}_{1}, t\right)= & \frac{8}{\sqrt{\pi}} \exp \left(-\frac{r_{1}^{2}}{a_{\mathrm{CM}}^{2}(t)}\right) \\
& \times \int_{0}^{\infty} d y y^{2} \exp \left(-\frac{y^{2}}{4}\right)\left|\psi_{000}^{\mathrm{RM}, 3 \mathrm{D}}\left(a_{\mathrm{CM}}(t) y, t\right)\right|^{2} \\
& \times \frac{\sinh \left(r_{1} y / a_{\mathrm{CM}}(t)\right)}{\left(r_{1} y / a_{\mathrm{CM}}(t)\right)}
\end{aligned}
$$

which constitutes a non-trivial generalization of the HHM result for the static ground state density given in Eq. (14) of that publication.

\section{Time-dependent atomic scattering factor}

A quantity which is easily accessible by experiment is the atomic scattering factor given by the Fourier transform of the atomic electron density

$$
f(\mathbf{k}, t)=\int n(\mathbf{r}, t) e^{i \mathbf{k r}} d \mathbf{r}
$$

Inserting Eq. (17) into Eq. (30) and taking advantage of the fact that the Jacobian for the transformation $\left\{\mathbf{r}_{1}, \mathbf{r}_{2}\right\} \rightarrow\{\mathbf{b}, \mathbf{c}\}$ is unity, we obtain

$$
\begin{aligned}
f_{\mathrm{tot}}(\mathbf{k}, t)= & 2\left[\int\left|\psi_{\mathrm{CM}}(\mathbf{c}, t)\right|^{2} e^{i \mathbf{k} \mathbf{c}} d \mathbf{c}\right] \\
& \times\left[\int\left|\psi_{\mathrm{RM}}(\mathbf{b}, t)\right|^{2} e^{i \frac{\mathbf{k b}}{2}} d \mathbf{b}\right] \\
:= & 2 f_{\mathrm{CM}}(\mathbf{k}, t) f_{\mathrm{RM}}(\mathbf{k} / 2, t),
\end{aligned}
$$

which shows that the total two-electron scattering factor $f_{\text {tot }}$ decouples into a product of one-particle scattering factors of the centre-of-mass and relative motion systems.

While the term $f_{\mathrm{RM}}$ remains until the relative motion Schrödinger equation is solved for a specific $\omega_{0}(t)$ and interparticle potential $u(b)$, the other piece can be evaluated based on the results of the last section. In three dimensions a closed solution is however only possible for the case of vanishing angular momentum. We therefore step back to the two dimensional problem studied by D'Amico and Vignale. For sake of simplicity we treat only the case for which the system is in an arbitrary eigenstate at $t=0$. The more general superposition of Eq. (10) can be solved along the lines of the following derivation and poses no additional problems. 
Using Eq. (12) the centre-of-mass structure factor reads

$$
f_{\mathrm{CM}}(\mathbf{k}, t) \equiv f_{\mathrm{CM}}(k, t)=\int_{0}^{\infty} J_{0}(k c)\left|\chi_{n m}(c, t)\right|^{2} c d c,
$$

where the angular integration led to the Bessel function of the first kind $J_{0}$. After insertion of Eq. (13) to (15) and polynomial expansion of the Laguerre functions according to

$$
L_{n}^{m}(x)=\sum_{s=0}^{n}\left(\begin{array}{c}
n+m \\
n-s
\end{array}\right) \frac{(-x)^{s}}{s !},
$$

we arrive at

$$
\begin{aligned}
f_{\mathrm{CM}}(k, t) & =\frac{n ! 2}{(n+m) !}\left[m_{\mathrm{cm}} \dot{\phi}(t)\right]^{m+1} \\
& \times \sum_{s=0}^{n} \sum_{t=0}^{n}\left(\begin{array}{c}
n+m \\
n-s
\end{array}\right)\left(\begin{array}{c}
n+m \\
n-t
\end{array}\right) \frac{\left(-m_{\mathrm{cm}} \dot{\phi}\right)^{s+t}}{s ! t !} \\
& \times \int_{0}^{\infty} J_{0}(k c) e^{-m_{\mathrm{cm}} \dot{\phi}(t) c^{2}} c^{2(m+s+t)+1} d c .
\end{aligned}
$$

The solution of the remaining integral is known [11] and involves the confluent hypergeometric functions of the Kummer type $M$ :

$$
\begin{aligned}
\int_{0}^{\infty} e^{-a^{2} t^{2}} t^{\mu-1} J_{\nu}(b t) d t= & \\
& \begin{aligned}
& \frac{\Gamma\left(\frac{\mu+\nu}{2}\right)\left(\frac{b}{2 a}\right)^{\nu}}{2 a^{\mu} \Gamma(\nu+1)} M\left(\frac{\mu+\nu}{2}, \nu+1,-\frac{b^{2}}{4 a^{2}}\right) \\
& \forall \quad \Re(\mu+\nu)>0 ; \quad \Re\left(a^{2}\right)>0 .
\end{aligned}
\end{aligned}
$$

The CM structure factor then takes the final form

$$
\begin{aligned}
f_{\mathrm{CM}}\left(k, m_{\mathrm{cm}} \dot{\phi}(t)\right)=\frac{n !}{(n+m) !} \sum_{s=0}^{n} & \sum_{t=0}^{n}\left(\begin{array}{c}
n+m \\
n-s
\end{array}\right)\left(\begin{array}{c}
n+m \\
n-t
\end{array}\right) \\
& \times \frac{\Gamma(m+s+t+1)}{s ! t !} M\left(m+s+t+1,1,-\frac{k^{2}}{4 m_{\mathrm{cm}} \dot{\phi}}\right),
\end{aligned}
$$

which constitutes one of the main results of this work. As indicated, the time-dependence of Eq. (36) is solely determined by the phase derivative $\dot{\phi}$, which equals the frequency $\omega_{0}$ of the confining harmonic potential in the static limit. For a system initially in its ground state, $f_{\mathrm{CM}}$ reduces to a simple Gaussian which correctly tends, respectively, towards the number of electrons (one in this case) as $k$ approaches zero, and zero as $k$ grows to infinity.

Having obtained a closed solution for the centre-of-mass structure factor we now briefly discuss the relative motion term. In general it will be necessary to evaluate this part by 
numerical methods, which is beyond the scope of the present article. The so-called Moshin-

sky atom [2] characterized by the harmonic interparticle potential $u(b)=-\frac{1}{2} \mathcal{K} b^{2}$ provides a useful exception and can be treated analytically. To this end we note that in this case the relative motion Hamiltonian is equivalent to a centre-of-mass one with effective mass $\tilde{m}_{\mathrm{cm}}=1 / 2$ and force constant $\omega_{0}^{2}(t)-\mathcal{K} / \tilde{m}_{\mathrm{cm}}$. Solving

$$
\tilde{X}(t)=|\tilde{X}(t)| e^{i \tilde{\phi}(t)} \quad \text { with } \quad \ddot{\tilde{X}}=-\left(\omega_{0}^{2}(t)-\frac{\mathcal{K}}{\tilde{m}_{\mathrm{cm}}}\right) \tilde{X},
$$

allows one to obtain the total structure factor of the Mochinsky atom from

$$
f_{\text {tot }}^{\mathcal{K}}(k, t)=2 f_{\mathrm{CM}}\left(k, m_{\mathrm{cm}} \dot{\phi}(t)\right) f_{\mathrm{CM}}\left(k / 2, \tilde{m}_{\mathrm{cm}} \dot{\tilde{\phi}}(t)\right) .
$$

\section{RELATION TO TDDFT}

As mentioned in the Introduction, this work was motivated by the serious criticism of Schirmer and Dreuw [6] of the 1984 theorems of Runge and Gross [12]. In Ref. [6] the proof in [12] was not only challenged but seemingly refuted. To be specific, the authors claimed that the variational derivation of the time-dependent Kohn-Sham equations in Ref. [12] is invalid due to an ill-defined action functional presented there. A nonvariational formulation would also run into problems, since in this case the Kohn-Sham system would allow one to reproduce but not to predict the exact electron density.

The results of this work can in principle be used to investigate this issue in an actual numerical calculation. For the two-electron spin-compensated system at hand, it is possible to construct the exact exchange-correlation potential from the known electron density in real (Eq. 29) or momentum space (Eq. 38). This is in line with van Leeuwen's proof [13] of mapping from densities to potentials in TDDFT with general two-particle interactions. Such a construction was for example already performed by Lein and Kümmel [14] or D'Amico and Vignale [9]. Using this potential in the numerical solution of the time-dependent Kohn-Sham orbitals would then open the opportunity to compare the propagated and exact electron density.

\section{SUMMARY AND POSSIBLE FUTURE DIRECTIONS}

As set out in the Introduction, HHM [1] proposed and solved the problem of a twoelectron spin-compensated family of harmonically confined atoms with a general interparticle 
repulsive potential $u\left(r_{12}\right)$.

Using the available time-dependent study of D'Amico and Vignale [9], but now in two dimensions, we display here the time-dependent density $n(\mathbf{r}, t)$ for a system initially in the ground state in three dimensions. While the general result is somewhat formal, for the so-called Moshinsky atom characterized by the choice $u(b)=-\frac{1}{2} \mathcal{K} b^{2}$ with $\mathcal{K}$ measuring the strength of the interparticle repulsion, we derive the momentum space electron density $f^{\mathcal{K}}(k, t)$ functionally from the D'Amico and Vignale form for the independent two-electron case with $\mathcal{K}$ set equal to zero.

For the future, progress may come by taking a specific choice of the time-dependence $\omega_{0}^{2}(t)$ of the harmonic confinement potential. Singling out a specific Fourier component through the choice $\exp i \omega t+\exp -i \omega t$ is restrictive to periodic time-dependence but may allow, in the future a more elegant discussion of the time-dependent particle density $n(\mathbf{r}, t)$ at the heart of all current theories of TDDFT. But, of course, more important would be to pass from a model 'He-like' family to a four-electron interacting 'Be-like' system, where an elegant Dirac idempotent density matrix already exists in the independent particle (Hartree-Fock or Kohn-Sham) limit (see, for example Refs. [15, 16]).

\section{ACKNOWLEDGEMENT}

NHM wishes to thank Professor A. Holas and I.A. Howard for invaluable discussions on the general area embraced by this study. Professor J. Schirmer is also thanked by NHM for allowing him early access to the material in Ref. [6]. TAN and NHM acknowledge a generous scholarship from the German Cancer Research Center, Heidelberg, which made their contribution to this work possible.

[1] A. Holas, I.A. Howard, and N.H. March, Phys. Lett. A 310, 451 (2003).

[2] M. Moshinsky, Am. J. Phys 36, 52 (1968).

[3] S. Kais, D.R. Herschbach, and R.D. Levine, J. Chem. Phys. 91, 7791 (1989).

[4] R. Crandall, R. Whitnell, and R. Bettega, Am. J. Phys. 52, 438 (1984).

[5] See also the very recent study of P. Capuzzi, N.H. March, and M.P. Tosi, J. Phys. A: Math and Gen. 38, L439 (2005). 
[6] J. Schirmer and A. Dreuw, Phys. Rev. A 75, 022513 (2007).

[7] A. Dreuw and M. Head-Gordon, Chem. Rev. 105, 4009 (2005).

[8] This goes back, at very least, to N.R. Kestner and O. Sinanoḡlu, Phys. Rev. 128, 2687 (1962), see also Ref. [3].

[9] I. DAmico and G. Vignale, Phys. Rev. B 59, 7876 (1999).

[10] The form of $A(t)$ given here differs by a factor of 2 from the result in Ref. [9].

[11] M. Abramowitz and I.A. Stegun, Handbook of Mathematical Functions (Dover, New York, 1964), p. 486.

[12] E. Runge and E.K.U. Gross, Phys. Rev. Lett. 52, 997 (1984).

[13] R. van Leeuwen, Phys. Rev. Lett. 82, 3863 (1999).

[14] M. Lein and S. Kümmel, Phys. Rev. Lett. 94, 143003 (2005).

[15] K.A. Dawson and N.H. March, J. Chem. Phys. 81, 5850 (1984).

[16] A. Holas, N.H. March, Y. Takahashi, and C. Zhang, Phys. Rev. A 48, 2708 (1993). 